See discussions, stats, and author profiles for this publication at: https://www.researchgate.net/publication/303278621

\title{
Passive seismic source localization via common-reflection-surface attributes
}

Article in Studia Geophysica et Geodaetica · May 2016

DOI: 10.1007/s11200-015-0493-x

\section{CITATIONS}

19

3 authors:

Benjamin Schwarz

1. Helmholtz-Zentrum Potsdam - Deutsches GeoForschungsZentrum GFZ 46 PUBLICATIONS 171 CITATIONS

SEE PROFILE

Dirk Gajewski

University of Hamburg

235 PUBLICATIONS 2,694 CITATIONS

SEE PROFILE

Some of the authors of this publication are also working on these related projects:

Imaging the subsurface with natural Green's functions View project

Project Unsupervised imaging and interpretation of seismic data View project
Alexander Bauer

University of Hamburg

19 PUBLICATIONS 59 CITATIONS

SEE PROFILE 


\title{
Passive seismic source localization via
}

\section{common-reflection-surface attributes}

\author{
Benjamin Schwarz, Alexander Bauer and Dirk Gajewski \\ Institute of Geophysics \\ University of Hamburg \\ Bundesstraße 55 \\ D-20146 Hamburg (Germany)
}

(March 14, 2017)

Keywords: CRS, stacking, moveout, localization, tomography

\begin{abstract}
The common-reflection-surface (CRS) stack can be viewed as a physically justified extension of the classical common-midpoint (CMP) stack, utilizing redundant information not only in a single but in several neighboring CMP gathers. The zero-offset CRS moveout is parameterized in terms of kinematic attributes, which utilize reciprocity and raypath symmetries to describe the two-way process of the actual wave propagation in active seismic experiments by the propagation of auxiliary one-way wavefronts. For the diffraction case, only the attributes of a single one-way wavefront, originating from the diffractor are sufficient to explain the traveltime differences observed at the surface. While paraxial ray theory gives rise to a second-order approximation of the CRS traveltime, many higher-order approximations were subsequently introduced either by squaring the second-order expression or by employing principles of optics and geometry. It was recently discovered that all of these higher-order operators can be formulated either for the optical projection or in an auxiliary
\end{abstract}


medium of a constant effective velocity. Utilizing this duality and the one-way nature of the CRS parameters, we present a simple data-driven stacking scheme that allows for the inversion of the a priori unknown excitation time of a passive seismic source. In addition, we demonstrate with a simple data example that the output of the suggested workflow can directly be used for subsequent focusing-based normal-incidence-point (NIP) tomography, leading to a reliable localization in depth. 


\section{INTRODUCTION}

Stacking still can be considered one of the most reliable processes in seismology and its origins date back to the late 60s (Mayne, 1962). In both, active and passive regimes, the coherent summation of amplitudes can lead to a significant increase of the signal-to-noise ratio and especially weak events, which were barely recognizable in the raw data can be made accessible for further processing and interpretation (Shearer, 1990). With the introduction of normalized velocity spectra (Taner and Koehler, 1969), the process of stacking can in principle be fully automized. The common-reflection-surface (CRS) stack is an extension of the classical common-midpoint (CMP) stack, in which many neighboring midpoints are jointly described by a traveltime surface (Jäger et al., 2001). While a decrease in the noise level is a virtue on its own, the estimated CRS operators can also be used for efficient prestack data enhancement, like trace interpolation or regularization (Baykulov and Gajewski, 2009). In addition, the CRS traveltime moveout expression is parameterized in terms of kinematic attributes of fictitious one-way propagation experiments (Hubral, 1983), which can be utilized for various applications, ranging from multiple suppression (Dümmong and Gajewski, 2008), diffraction separation (Dell and Gajewski, 2011), geometrical spreading compensation (Hubral, 1983), Fresnel zone estimation for migration (Hubral et al., 1993) to advanced depth velocity model building via NIP tomography (Duveneck, 2004).

Although originally, a second-order expression followed from the propagator formalism by Bortfeld (1989) in paraxial ray theory, a hyperbolic operator is commonly favored, since it proved to provide higher accuracy in the presence of predominantly vertical heterogeneity

(Schleicher et al., 1993). Alongside the co-existing multifocusing approach and the esti- 
mation of local event slopes (Fomel, 2007), several higher-order CRS formulations have been introduced in recent years (Landa et al., 2010; Fomel and Kazinnik, 2013; Schwarz et al., 2014b). By extending the work of de Bazelaire (1988) and Höcht et al. (1999), Schwarz et al. (2014a, 2015) demonstrated that all these higher-order expressions, including the commonly used hyperbolic CRS operator, can either be formulated for the optical projection or an effective analog of the true kinematics of the wave propagation. In these different domains, heterogeneity is accounted for either by a shift in time or in velocity. In addition, they provided an alternative CRS parameterization, in which dip, curvature and heterogeneity effects are handled by separate attributes. In that frame, they introduced a CRS-type extension of de Bazelaire's osculating equation, which allows for a free transformation between the optical and the effective domain.

While in active seismic acquisitions, the location and the excitation time of the source is generally known, the processing is primarily concerned with resolving and inverting the structure and properties of the traversed medium (Sheriff and Geldart, 1995). A passive seismic problem, in contrast, faces the additional difficulties of unknown source characteristics. Closing the gap between traditional large-scale earthquake seismology and controlledsource seismic exploration, Rutledge and Phillips (2003) found that microseismicity can be utilized to image the process of the stimulation of hydraulic fracturing. Besides its essential use in the imaging of low-amplitude phases like precursors on continental or even global scales (Shearer, 1990), stacking proved to be also a useful tool for the localization and characterization of microseismic events (Gajewski et al., 2007; Zhebel et al., 2011). Khoshnavaz et al. (2015) showed, that local slopes alone can already be used for successful passive event localization, if the overburden complexity can be considered moderate. Though, their 
method cannot account for strong overburden heterogeneity and does not provide information on the velocity distribution in depth. Following from symmetry principles, the CRS attributes describe actual two-way propagation by an auxiliary one-way kinematic point source experiment. In this work, we introduce a passive seismic stacking operator, that is formulated in terms of the CRS attributes. By utilizing the aforementioned duality of higher-order moveouts, we suggest a simple strategy to extract the a priori unknown excitation time of a passive seismic event and propose the subsequent use of NIP tomography (Duveneck, 2004) to jointly invert for the depth location and the overburden velocity model. A synthetic example illustrates that in contrast to waveform-based joint location and velocity inversion approaches (Kamei and Lumley, 2014), the presented method has the potential to perform sufficiently stable even in noisy environments and does not require detailed a-priori velocity information.

\section{THEORY}

\section{Common-reflection-surface attributes and diffractions}

[Figure 1 about here.]

The common-reflection-surface (CRS) stack (Jäger et al., 2001) can be viewed as a generalization of the classical CMP stack method (Mayne, 1962), in which the coherent summation follows a moveout surface, which extends over several neighboring CMP gathers. Consequently, in the 2D case, the zero-offset CRS stacking operator depends on three parameters, which have to be estimated via coherence analysis. Despite its higher dimensionality, the CRS moveout is expressed in terms of surface-related kinematic wavefield attributes, which exploit raypath symmetries to describe the general two-way reflection kine- 
matics by two fictitious one-way propagating wavefront experiments (Hubral, 1983). For the diffraction case, both wavefronts coincide (Dell and Gajewski, 2011) and the observed traveltime differences can be fully described by attributes of the emerging NIP wavefront. In slowness notation, the zero-offset diffraction subset of the conventional hyperbolic CRS moveout (Schleicher et al., 1993; Jäger et al., 2001) can be written as

$$
\Delta t\left(t_{0}, p_{0 x}, p_{\text {shift }}\right)=\sqrt{\left(t_{0}+2 p_{0 x} \Delta x_{m}\right)^{2}+4\left(p_{\text {shift }}^{2}-p_{0 x}^{2}\right) \Delta x_{m}^{2}}-t_{0}
$$

with the effective slowness $p_{\text {shift }}$ and the true horizontal slowness of the emerging NIP wave $p_{0 x}$ defined according to

$$
\begin{aligned}
p_{0 x} & =\frac{\sin \alpha_{0}}{v_{0}}, \\
p_{\text {shift }} & =\frac{\sqrt{1+\left(v_{\mathrm{NMO}} / v_{0}\right)^{2} \sin ^{2} \alpha_{0}}}{v_{\mathrm{NMO}}},
\end{aligned}
$$

where

$$
v_{\mathrm{NMO}}=\sqrt{\frac{2 v_{0} R_{\mathrm{NIP}}}{t_{0} \cos ^{2} \alpha_{0}}}
$$

is the normal moveout (NMO) velocity and $v_{0}$ denotes the locally constant near-surface velocity, i.e. the velocity at the acquisition line near the surface. As follows from equation (1), the presented conventional CRS parameterization implies that the moveout $\Delta t$ is a function of the reference traveltime $t_{0}$, which corresponds to the actual propagation traveltime 
of the zero-offset reference ray emerging at the central trace location $x_{0}$ (compare Figure 1). The quantity $\Delta x_{m}=x_{m}-x_{0}$ denotes the lateral separation of every trace within the considered aperture with respect to its central reference trace. Following from reciprocity, Hubral (1983) concluded that in order to accurately describe the moveout of reflected events, the conceptual NIP wave, illustrated in Figure 1 must travel at half the medium velocity. For the actual true velocity distribution, accordingly, a seismic point source placed at the normal-incidence-point would take $t_{0} / 2$ to arrive at the central midpoint location at the surface. In the next subsection, we will briefly review the duality of higher order CRS-type moveouts in order to introduce a generalized passive seismic moveout approximation.

\section{A generalized passive seismic moveout}

[Figure 2 about here.]

As emphasized before, the fictitious concept of the NIP wave, utilizing raypath symmetries and reciprocity, proved to be very useful in reducing the complex two-way ray geometry of a reflection experiment to the much simpler geometry of a one-way propagating wavefront, which leads to the same description of the moveout (Hubral, 1983). For a passive experiment, this one-way experiment is physically real and the fictitious source can in this case be identified with the true source of the passive event. Due to the coincidence of the up and down-going ray paths in the zero-offset section, the moveout of a one-way propagating wave follows from halving the active source CRS diffraction operator (1),

$$
\Delta t=\sqrt{\left(t_{0}+p_{0 x} \Delta x_{g}\right)^{2}+\left(p_{\text {shift }}^{2}-p_{0 x}^{2}\right) \Delta x_{g}^{2}}-t_{0}
$$


in which the midpoint displacement $\Delta x_{m}$ is replaced by the relative lateral receiver distance $\Delta x_{g}=x_{g}-x_{0}$ and $t_{0}$ now denotes the one-way traveltime from the NIP to the central receiver in the aperture. Formally, as stressed before, this expression is exactly equivalent to the diffraction case in the zero-offset configuration, with the only difference that for the active experiment the NIP wave, per definition, travels at half the actual medium velocity, whereas in the considered passive scenario, the propagation takes place at the actual velocity.

As mentioned before, the CRS moveout in its conventional parameterization depends on the zero-offset reference time $t_{0}$ (compare equation (1)). Following the works of de Bazelaire (1988) and Höcht et al. (1999), Schwarz et al. (2014a, 2015) found that all higher-order CRS-type stacking operators, including multifocusing (Gelchinsky et al., 1999), can be formulated in terms of the CRS attributes in two different ways, one accounting for overburden heterogeneity by the application of a constant slowness shift, the other, in correspondence with de Bazelaires shifted hyperbola, by a constant shift of the reference time. According to Höcht et al. (1999) and Schwarz et al. (2014b), both mechanisms can be related to the CRS attributes via the relations (2) and

$$
t_{\text {shift }}=\frac{R_{\mathrm{NIP}}}{v_{0}}
$$

which corresponds to the definition of the shifted passive seismic moveout reference time. Schwarz et al. (2015) found that slowness shifts can be transformed into time shifts and vice versa via the generalized osculating equation

$$
p_{\text {shift }}^{2}=p_{0 x}^{2}+\frac{t_{0}}{t_{\text {shift }}}\left(p_{0}^{2}-p_{0 x}^{2}\right)
$$


where, for convenience, $p_{0}=1 / v_{0}$ denotes the slowness near the surface. The ray parameter near the surface $p_{0 x}$, which is related to the CRS attributes via (2) describes the local event dip in the recorded data. It can be formally shown that all CRS-type operators of higher order than two can be transformed to their time-shifted representation by applying the osculating condition (6). By re-parameterization according to equation (6), which is equally valid for the active and for the passive seismic case, the previously introduced moveout (4) can either be formulated with a time shift or a slowness shift mechanism to account for overburden heterogeneity. Both versions of the passive traveltime moveout can thus be written as one generalized equation

$$
\begin{aligned}
t & =t_{s}+t_{0}+\Delta t\left(\hat{t}_{0}, \hat{p}\right) \\
& =t_{s}+t_{0}+\sqrt{\left(\hat{t}_{0}+p_{0 x} \Delta x_{g}\right)^{2}+\left(\hat{p}^{2}-p_{0 x}^{2}\right) \Delta x_{g}^{2}}-\hat{t}_{0}
\end{aligned}
$$

where, depending on the choice of the user, $\left(\hat{t}_{0}, \hat{p}\right)$ can either be replaced by $\left(t_{\text {shift }}, p_{0}\right)$ to estimate wavefront curvature and dip, or by $\left(t_{0}, p_{\text {shift }}\right)$ to include the total reference traveltime in the approximation. The time shift and the slowness shift approach are compared in a conceptual sketch shown in Figure 2. While the moveouts, due to the aforementioned symmetries, are very similar for the active zero-offset case and a passive setting, the main difference constitutes in the fact that the excitation time of the passive source, denoted by $t_{s}$ is generally not known for the second case. In the following, we suggest a hybrid scheme, in which subsets of the generalized operator (7) are used to sequentially measure the attributes $p_{0 x}, t_{\text {shift }}$ and $t_{0}$, the latter allowing a direct moveout-based inversion of the source time $t_{s}$. As will be shown subsequently, the correction of the respective constant 
event source time will allow for wavefront-based NIP tomography (Duveneck, 2004) to be performed to achieve a simultaneous depth localization and overburden velocity inversion.

\section{WORKFLOW AND DATA EXAMPLE}

[Figure 3 about here.]

[Figure 4 about here.]

Following the introduction of the passive seismic strategy, we will demonstrate the principal applicability of the suggested scheme to a passive seismic dataset. From a kinematic viewpoint, a passive seismic experiment is characterized by the additional complication of the unknown source time. Thus, we simulate a passive seismic experiment by modeling the response of three buried seismic sources in a vertically and laterally heterogeneous background and subsequently shift the recorded events by an arbitrary amount in time. The chosen simulated excitation times of the three sources, as well as their true locations and the velocity structure in depth are displayed in Figure 4. Figure 5 shows the modeled data with a trace spacing of $25 \mathrm{~m}$ and a dominant event frequency of $30 \mathrm{~Hz}$ (chosen for convenience) without time delays applied (left) and the simulated passive acquisition with time-shifts applied (right). In addition, we chose to add Gaussian random noise with a signal-to-noise ratio of 5 to the data, to ensure a more realistic setting. The chosen receiver apertures vary from $300 \mathrm{~m}$, for the initial dip search, to $1000 \mathrm{~m}$ and $2000 \mathrm{~m}$ for the curvature-related measurements of the shifted reference and the source time. While, for convenience, the initial one-parameter searches constitute in fine-meshed grid searches, parameters are subsequently refined using a Nelder Mead simplex optimization algorithm (Nelder and Mead, 1965). As illustrated in Figure 3, the initial CRS parameter estimation is followed by an 
automated correction for the estimated source times, whose results are then fed into the NIP tomographic inversion scheme by Duveneck (2004).

\section{Attribute estimation}

[Figure 5 about here.]

In the following, we suggest a simple and stable scheme to sequentially measure and refine all unknowns in equation (7) via redundancy-exploiting multi-parameter coherence analysis. The measure of coherence, in accordance with current implementations of the CRS stack and similar to conventional velocity analysis is the normalized semblance coefficient (Taner and Koehler, 1969). The first step of this strategy is equivalent to the plane wave search suggested for the pragmatic CRS approach (Jäger et al., 2001) and makes use of the first-order subset of the generalized passive operator (7)

$$
\Delta t \approx \Delta t\left(p_{0 x}\right)=p_{0 x} \Delta x_{g}
$$

Since the slope attribute $p_{0 x}$, i.e. the local traveltime dip, is independent of the choice of the auxiliary medium, i.e. the optical projection space and the effective medium, the actual reference traveltime and the source excitation time both do not need to be known for this first measurement. The second step of the suggested pragmatic approach consists of a second-order moveout measurement for the optical projection of the problem, i.e. the time-shift mechanism. According to Schwarz et al. (2014a), time-shifted moveouts in general as well do not depend on the actual reference time and therefore can also be accurately measured without the knowledge of $t_{0}$ and $t_{s}$ 


$$
\Delta t=\Delta t\left(p_{0 x}, t_{\text {shift }}\right)=\sqrt{\left(t_{\text {shift }}+p_{0 x} \Delta x_{g}\right)^{2}+\left(p_{0}^{2}-p_{0 x}^{2}\right) \Delta x_{g}^{2}}-t_{\text {shift }}
$$

where $p_{0}=1 / v_{0}$ is the inverse of the near-surface velocity, i.e. the near-surface slowness at the receiver. Since the horizontal slowness was gained in the preceding dip measurement (8), this second step, based on equation (9), consists again of a one parameter search, in this case for the shifted reference time $t_{\text {shift }}$.

[Figure 6 about here.]

While until now, only the time-shift mechanism was utilized, the third and final step of the suggested pragmatic scheme makes use of the second face, i.e. the slowness-shifted version of the passive moveout operator (7). In the considered frame of a passive seismic experiment, this dependency of the slowness-shifted moveout on the actual reference time $t_{0}$ proves to be very valuable and allows for the inversion of the source excitation time $t_{s}$, which needs to be corrected before NIP tomography can be applied. We have, again based on the preceding measurements of $p_{0 x}$ and $t_{\text {shift }}$, a one-parameter optimization problem

$$
\begin{aligned}
t & =t_{s}+t_{0}+\sqrt{\left(t_{0}+p_{0 x} \Delta x_{g}\right)^{2}+\left[p_{\text {shift }}^{2}\left(t_{0}\right)-p_{0 x}^{2}\right] \Delta x_{g}^{2}}-t_{0} \\
& =t_{s}+\sqrt{\left(t_{0}+p_{0 x} \Delta x_{g}\right)^{2}+\left[p_{\text {shift }}^{2}\left(t_{0}\right)-p_{0 x}^{2}\right] \Delta x_{g}^{2}}
\end{aligned}
$$

with $p_{\text {shift }}$ defined via the generalized osculating equation, which, as mentioned before, is 
equally valid for active and passive seismic experiments.

Figure 6 shows the estimated attributes for the chosen synthetic example following the suggested scheme, which constitutes of determining

1. the horizontal slowness $p_{0 x}$ using moveout (8),

2. the shifted reference time $t_{\text {shift }}$ using moveout (9), and

3. the source time $t_{s}$ using the total traveltime (10).

While due to the added noise level, the passive events are not clearly visible over the full considered receiver line, in the attributes sections the coherent energy is well recognizable. The consistently high coherence values along the events, shown in Figure 6(a), indicate the appropriate fit of the passive operator in the considered aperture range (here displayed for the one-parameter $t_{\text {shift }}$ search). Being a local measurement, the slope estimation, which is shown in Figure 6(b), performed reasonably stable and resulted in the expected smooth behavior along the events. In addition, the large range of slope values indicates the good ray coverage of point-like passive seismic events, illuminating large portions of the underlying velocity model (compare Figure 4). Being related to the wavefront curvature measured at the surface, the shifted reference traveltime and the source excitation time need to be estimated in larger apertures. Nevertheless, the corresponding attribute panels shown in Figure 6(c) and 6(d) reveal a generally smooth distribution of values along the events. Especially the source times are estimated with reasonable accuracy, showing a very good correspondence with the exact values of $0 \mathrm{~s}, 5 \mathrm{~s}$, and $10 \mathrm{~s}$. It can be concluded that for the considered synthetic example, the proposed strategy allows for a robust fully automatic 
estimation not only of the dip and curvature moveout contributions, but also for a reasonably accurate inversion of the unknown excitation times. As will be demonstrated in the following, every passive event has a characteristic source time and therefore allows for a data-driven correction of the individual events.

[Figure 7 about here.]

\section{Joint localization and velocity inversion}

[Figure 8 about here.]

Due to the formal equivalence of the fictitious NIP wave and the true passive experiment, The extracted attributes can directly be used for efficient localization and joint velocity inversion in depth. The conventional inversion according to Duveneck (2004) needs the emergence angle $\alpha_{0}$, the emerging NIP wave radius $R_{\mathrm{NIP}}$ and the true propagation time of the reference ray $t_{0}$ as input. As a result, the locations of the individual normal-incidence-points and the traversed overburden velocity structure are inverted and can be jointly displayed for interpretational purposes. In the available implementation the picking of locally coherent contributions is performed fully automatically using the coherence and stacked sections. In order to be performed, NIP tomography requires, as mentioned before, the knowledge of the true propagation time of the respective reference rays. Thus, the estimated passive attributes $\left(p_{0 x}, t_{\text {shift }}\right)$ need to be transformed to $\left(\alpha_{0}, R_{\mathrm{NIP}}\right)$ using equations $(2)$ and $(5)$ and, in addition, all required input sections must be corrected for the individual estimated event source time $t_{s}$, since it is not related to the actual wavefront propagation and, thus, does not contain information on the subsurface velocity distribution. Since NIP tomography is commonly used on two-way reflection data, an additional factor of two has to be applied 
on the time axes. Figure 7 shows the respective transformed and corrected sections for the considered synthetic dataset. As can be observed the source time estimation and correction was reasonably successful in reproducing the raw modeled data displayed on the right side of Figure 5 .

The near-surface velocity of $v_{0}=2000 \mathrm{~m} / \mathrm{s}$ was used as the starting velocity model for NIP tomography. In Figure 8(a) the corresponding NIP localization results are superimposed on the velocity model. The individual NIP locations do not correspond with the actual source positions denoted by the three circles. Figure 8(b) shows the result of NIP tomographic inversion, where the NIP locations correspond to the locally estimated positions of the passive sources. The suggested data-driven strategy based on the CRS attributes not only leads to a reasonable localization, it also jointly provides an estimate of the velocity model in depth. Comparison with the true model shown in Figure 4 reveals that the presented scheme is capable to successfully resolve the general velocity structure traversed by the measured passive seismic events.

\section{DISCUSSION}

Following the common-reflection-surface stacking methodology, the proposed scheme does not require any user-defined constraints or manual picking in the attribute estimation. While in the presented syntehtic example, the total recording times can be considered already very close to the actual traveltimes, continuous data recordings make a full threeparameter search for every recorded time sample very expensive. The suggested three-step strategy is not only aimed at stabilizing the attribute estimation, but also helps to decrease the computational costs, since after a highly parallel and efficient local slope estimation for the full data, the considerably more expensive curvature and excitation time estimation can 
be confined to data portions exhibiting sufficiently high coherence. The localization procedure can be divided into two parts, the first one consisting in estimating emerging wavefront attributes in the data itself, the second dealing with the inversion, i.e. back-propagation of the measured wavefronts into the subsurface (compare Figure 3). The central idea is that the actual reference time $t_{0}$, which denotes the time the excited wavefront needed to travel from the unknown source location to the central receiver location of the considered aperture is intrinsically encoded in the conventional hyperbolic CRS diffraction operator. This means that, in principle, the passive wavefront's excitation time can be accurately determined via coherence-based moveout analysis, i.e. only through analyzing the event's traveltime differences observed at the registration surface. Following from this, the source excitation time $t_{s}$ is just estimated once in the coherence-based moveout analysis and is kept fixed throughout the whole depth inversion procedure, which is essentially identical to the conventional NIP tomography commonly applied in the context of active reflection surveys. In correspondence, the depth inversion and the data-domain local wavefront attribute estimation are fully separated in the suggested passive localization scheme. In the context of reservoir monitoring, the accurate knowledge of the excitation times of actively controlled perforation shots can be used to directly and reliably invert for the velocity model and might help to sufficiently constrain the traveltime searches of following passively induced events.

As most other tomographic inversions, the accuracy and resolution of the inverted velocity models depends on the ray coverage, and, therefore also on the distribution of sources. Since, however, the suggested moveout-based localization and velocity inversion is performed for every receiver trace, this method makes use of the full registered angle coverage, which helps to invert velocity structure also near the boundaries of the acquisition. A gen- 
erally favored assumption of CRS-type moveout analysis is that the so-called near-surface velocity $\left(v_{0}\right)$ distribution, i.e. the velocity field at the recording receivers is known. For the presented simple example, we made this assumption of an a priori known constant value of $2000 \mathrm{~m} / \mathrm{s}$ for the near-surface velocity to constrain the inversion. Despite the simple example, the near-surface velocity is only required to be constant locally, on the scale of the considered apertures. It is important to note that, similar to the conventional CRS stack used in active seismic context, a general prerequisite of this type of moveout analysis is the preceding application of statics corrections.

The described method naturally requires a good ray coverage in order to provide a reasonably accurate inversion result for the velocity distribution. NIP tomography can be considered a moveout-constrained, focusing-based transmission tomography and as a result, it can only deliver trustworthy velocities for portions of the model, which were traversed by the recorded seismic energy. The full treatment of phase characteristics and source mechanisms appears hardly possible in the CRS framework. The presented scheme, we feel, takes its strength from the fact that it is a local approach, i.e. that it performs many local localizations and velocity inversions for a single recorded event (depending on the chosen aperture sizes). Despite the fact that we only considered simple explosive sources in the synthetic study, this means that the method, in principle, only requires waveforms to be locally consistent, i.e. of the same polarity, in order to be applicable. As a result, the event's apex (in contrast to other diffraction stacks) does not necessarily have to be recorded in order to allow for a successful localization and the method can likewise also be applied to monotypic S-wave data. In addition, the multiple localizations per event can be used to derive statistical means quantitatively reflecting the reliability of the results. Nevertheless, in its current form the proposed scheme is of limited use, when the direct inversion of i.e. 
the source radiation pattern is desired.

\section{CONCLUSIONS AND OUTLOOK}

We have presented a fully data-driven stacking scheme for passive seismic event localization in depth, which makes direct use of the formal equivalence of the NIP wave experiment and the actual excitation of a real passive seismic source. Based on a higher-order duality of CRS-type moevoeut approximations, we introduced the 2D passive seismic analog of a generalized diffraction hyperbola, whose application allows for the local estimation of wavefront tilt, curvature and the excitation time of a passive seismic event. By correcting for the estimated source time, the resulting kinematic attributes can directly be fed into the NIP tomographic scheme, which was successfully applied in the context of controlled source data acquisitions with a high data redundancy. To demonstrate the feasibility of the suggested approach, we applied this generalized passive stacking operator to a simple synthetic dataset simulating the sequential recording of three passive seismic events, whose excitation times were assumed to be unknown. The time-shifted version of the passive moveout was shown to be independent of the central ray traveltime, which likewise makes it applicable to active and passive seismic data, the velocity-shifted counterpart allows for a moveout-based inversion of the unknown source time. In the presented data example, the joint application of both moveout formulations led to reasonable estimates of the kinematic wavefield attributes for all three considered events. Starting from a generic constant background, the subsequent application of NIP tomography converged quickly and not only led to a robust event depth localization but it was also able to reasonably recover the heterogeneous velocity structure of the overburden. 
A robust estimation of the source excitation time proved to be crucial for a successful localization in depth. Since the presented methodology utilizes a moveout duality, which only shows in orders higher than two, the respective search apertures must be chosen reasonably large, which for complex heterogeneous models may degrade the local character, and therefore, the resolving power of the presented data-driven localization and inversion strategy. Besides the obvious need for an extension to three dimensions, a refinement of the source time estimation will be key to ensure the subsequent applicability of NIP tomography. Since in the context of reservoir monitoring, perforation shots are actively controlled and the respective source excitation times are known, future work will also deal with quantifying the reliability and accuracy of the proposed moveout-based source time estimation strategy in complicated field settings. In addition, the influence of the ray coverage on the accuracy and reliability of the location and velocity inversion should be thoroughly investigated. The current implementation of NIP tomography (Duveneck, 2004) allows for a locally independent localization, which in active seismic applications is a reasonable assumption, since for reflections, following Huygens principle, a single event is usually described by a collection of neighboring fictitious point source experiments. In the passive scenario, however, very much like for the diffraction case, an individual event can be related to a single point source (or focus) in depth. The knowledge of the source excitation time is an attribute that globally characterizes a passive event and, therefore, can be used to substantially constrain the NIP tomographic scheme, provided the assumption of a point source is reasonably justified. In addition, the utilized formal correspondence of the active seismic NIP wave concept and the passive seismic ray geometry suggests combined active and passive seismic applications, based on the same simple theoretical framework. 


\section{ACKNOWLEDGMENTS}

This work was kindly supported by the sponsors of the Wave Inversion Technology (WIT)

Consortium, Hamburg, Germany, and is part of the project Imaging steep structures with diffractions funded by the German Federal Ministry of Economic Affairs and Energy (BMWi

0325363C). Norsar 3D was used to generate the presented data example. We thank Petr Bulant and the two anonymous reviewers for valuable suggestions to improve the paper. 


\section{REFERENCES}

Baykulov, M., and D. Gajewski, 2009, Prestack seismic data enhancement with partial common-reflection-surface (CRS) stack: Geophysics, 74, V49-V58.

Bortfeld, R., 1989, Geometrical ray theory: Rays and traveltimes in seismic systems (secondorder approximations of the traveltimes): Geophysics, 54, 342-349.

de Bazelaire, E., 1988, Normal moveout revisited - inhomogeneous media and curved interfaces: Geophysics, 53, 143-157.

Dell, S., and D. Gajewski, 2011, Common-reflection-surface-based workflow for diffraction imaging: Geophysics, 76, S187-S195.

Dümmong, S., and D. Gajewski, 2008, A multiple suppression method via CRS attributes: Presented at the SEG Expanded Abstracts, Society of Exploration Geophysicists.

Duveneck, E., 2004, Velocity model estimation with data-derived wavefront attributes: Geophysics, 69, 265-274.

Fomel, S., 2007, Velocity-independent time-domain seismic imaging using local event slopes: Geophysics, 72, S139-S147.

Fomel, S., and R. Kazinnik, 2013, Non-hyperbolic common reflection surface: Geophysical Prospecting, 61, 21-27.

Gajewski, D., D. Anikiev, B. Kashtan, E. Tessmer, and C. Vanelle, 2007, Seg technical program expanded abstracts 2007.

Gelchinsky, B., A. Berkovitch, and S. Keydar, 1999, Multifocusing homeomorphic imaging - part 1. Basic concepts and formulae: Journal of Applied Geophysics, 42, 229-242.

Höcht, G., E. de Bazelaire, P. Majer, and P. Hubral, 1999, Seismics and optics: hyperbolae and curvatures: Journal of Applied Geophysics, 42, 261-281.

Hubral, P., 1983, Computing true amplitude reflections in a laterally inhomogeneous earth: 
Geophysics, 48, 1051-1062.

Hubral, P., J. Schleicher, M. Tygel, and C. Hanitzsch, 1993, Determination of Fresnel zones from traveltime measurements: Geophysics, 58, 703-712.

Jäger, R., J. Mann, G. Höcht, and P. Hubral, 2001, Common-reflection-surface stack: Image and attributes: Geophysics, 66, 97-109.

Kamei, R., and D. Lumley, 2014, Passive seismic imaging and velocity inversion using full wavefield methods: Presented at the 2014 SEG Annual Meeting, Society of Exploration Geophysicists.

Khoshnavaz, M. J., K. Chambers, A. Bóna, and M. Urosevic, 2015, Passive Seismic Localization Without Velocity Model: Application and Uncertainty Analysis: Presented at the 2015 SEG Annual Meeting, Society of Exploration Geophysicists.

Landa, E., S. Keydar, and T. J. Moser, 2010, Multifocusing revisited - inhomogeneous media and curved interfaces: Geophysical Prospecting, 58, 925-938.

Mayne, W. H., 1962, Common reflection point horizontal data stacking techniques: Geophysics, 27, 927-938.

Nelder, J. A., and R. Mead, 1965, A simplex method for function minimization: The computer journal, 7, 308-313.

Rutledge, J. T., and W. S. Phillips, 2003, Hydraulic stimulation of natural fractures as revealed by induced microearthquakes, Carthage Cotton Valley gas field, east Texas: Geophysics, 68, 441-452.

Schleicher, J., M. Tygel, and P. Hubral, 1993, Parabolic and hyperbolic paraxial two-point traveltimes in 3D media: Geophysical Prospecting, 41, 495-513.

Schwarz, B., C. Vanelle, and D. Gajewski, 2014a, Auxiliary Media - A Generalized View on Stacking: Presented at the 76th EAGE Conference and Exhibition, European Association 
of Geoscientists and Engineers.

, 2015, Shifted Hyperbola Revisited - The Two Faces of NMO: Presented at the 77th EAGE Conference and Exhibition, European Association of Geoscientists and Engineers.

Schwarz, B., C. Vanelle, D. Gajewski, and B. Kashtan, 2014b, Curvatures and inhomogeneities: An improved common-reflection-surface approach: Geophysics, 79, S231-S240.

Shearer, P. M., 1990, Seismic imaging of upper-mantle structure with new evidence for a 520-km discontinuity: Nature, 344, 121-126.

Sheriff, R. E., and L. P. Geldart, 1995, Exploration seismology: Cambridge university press.

Taner, M. T., and F. Koehler, 1969, Velocity spectra - digital computer derivation applications of velocity functions: Geophysics, 34, 859-881.

Zhebel, O., D. Gajewski, and C. Vanelle, 2011, Localization of seismic events in 3D media by diffraction stacking: Presented at the 73rd EAGE Conference \& Exhibition, European Association of Geoscientists and Engineers. 


\section{LIST OF FIGURES}

1 Illustration of the NIP wave concept . . . . . . . . . . . . . 25

2 Higher-order moveout duality . . . . . . . . . . . . . . . . 26

3 Passive seismic flowchart . . . . . . . . . . . . . . . . . . 27

$4 \quad$ Velocity model used for modeling . . . . . . . . . . . . . . . . . . . . 28

$5 \quad$ Modeled and simulated passive data . . . . . . . . . . . . . . . . 29

$6 \quad$ Attribute estimation results . . . . . . . . . . . . . . . . . 30

$7 \quad$ Corrected attribute sections suited for NIP tomography . . . . . . . . . . . 31

8 Joint localization and inversion via NIP tomography . . . . . . . . . . . . 32 


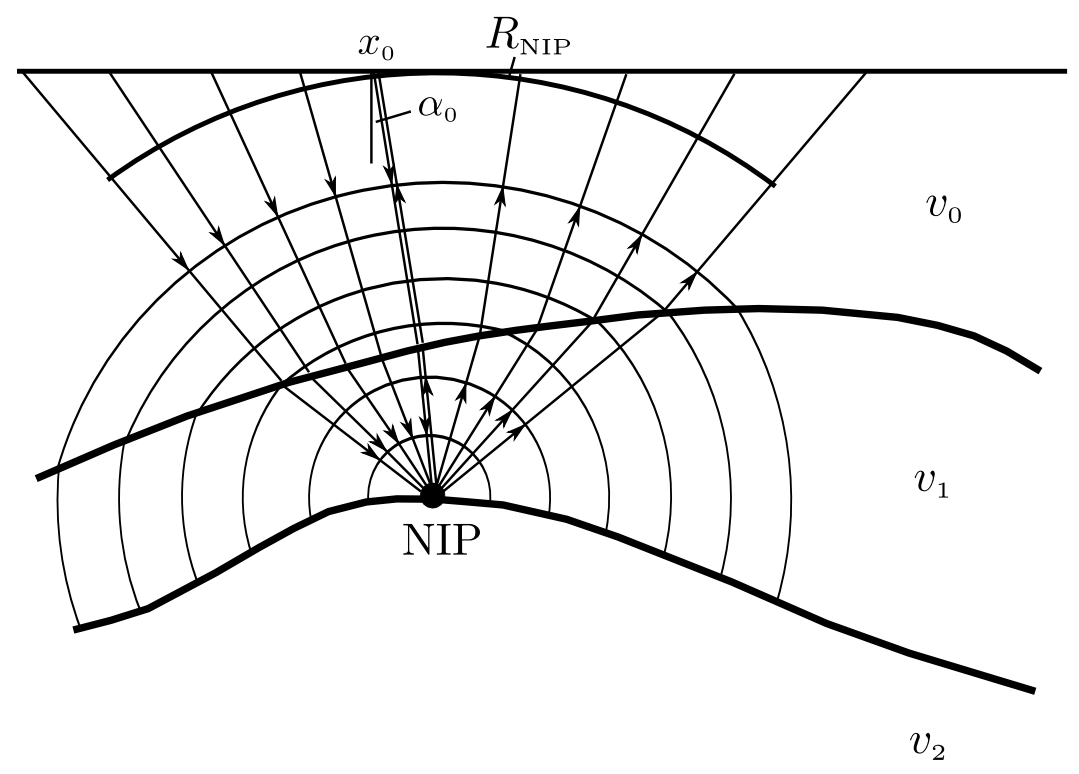

Figure 1: Based on the reciprocity of ray paths, the normal-incidence-point (NIP) can be viewed as a fictitious source in depth (Hubral, 1983). For Snell's law to be fulfilled only certain emission angles are realized and thus, for a general reflection, the source and receiver angles are coupled. For a diffraction or an actual passive seismic source in depth, the acquisition coordinates are decoupled and the NIP wave's emergence angle $\alpha_{0}$ and its emerging wavefront radius $R_{\mathrm{NIP}}$ are sufficient to describe the traveltime moveout observed at the surface. 
Receiver coordinate

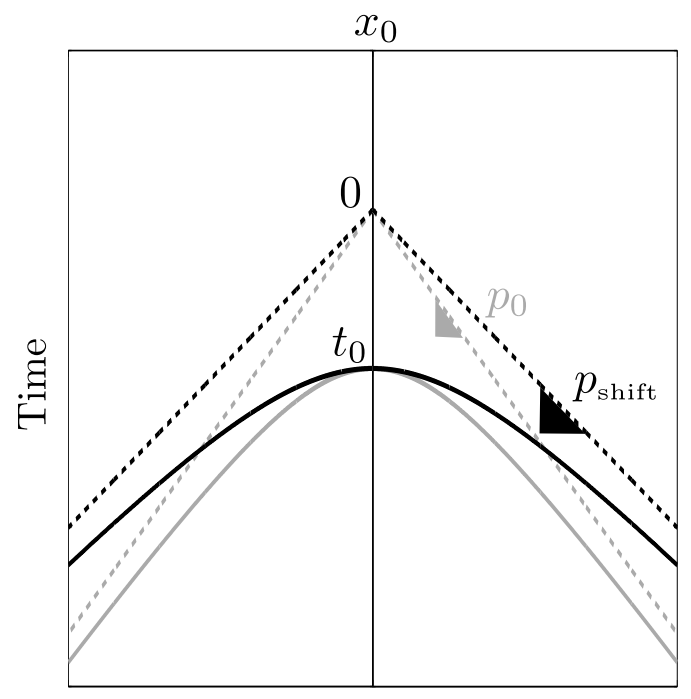

(a) Velocity shift
Receiver coordinate

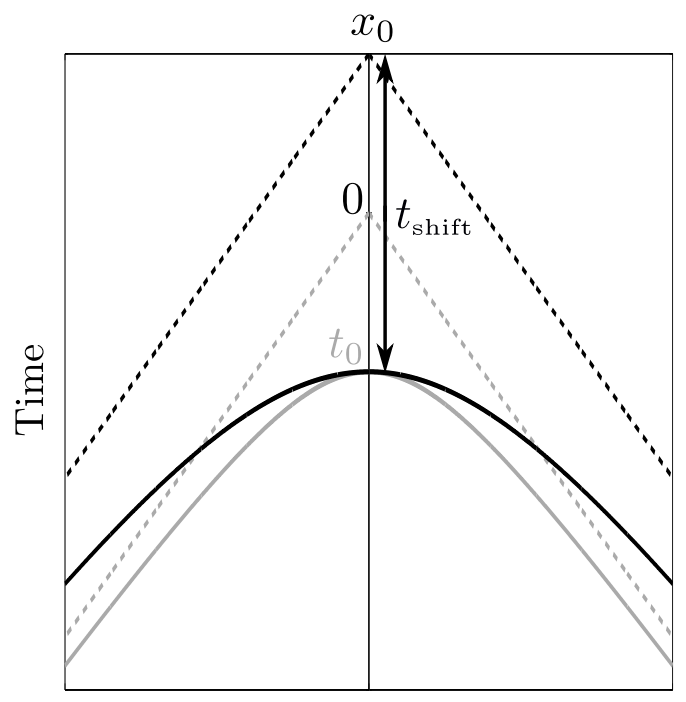

(b) Time shift

Figure 2: The two different ways to change the suggested passive seismic moveout expression to account for heterogeneity: (a) Classical velocity spectra approach, where the slowness $p_{\text {shift }}$ is perturbed (Taner and Koehler, 1969). (b) Application of a time shift $t_{\text {shift }}$ (de Bazelaire, 1988). Adapted traveltime curves are in bold lines, their respective asymptotes are dotted. Both shifts lead to a comparable perturbation of moveout (indicated in bold black). The unperturbed moveout is shown in gray. 


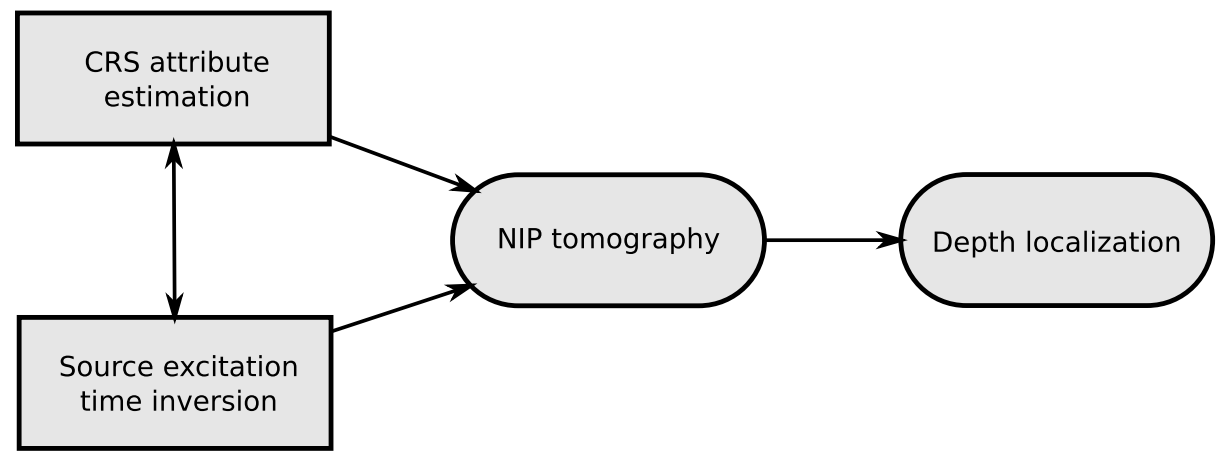

Figure 3: Overview of the suggested passive seismic workflow. In the first stage, the horizontal slopes and time shifts, which can be translated into the CRS parameters $\alpha_{0}$ and $R_{\mathrm{NIP}}$, are estimated using the time-shifted version of the passive operator. Subsequently, the effective medium moveout is applied to gain the source excitation time $t_{s}$ of the respective passive event. After correcting for the source time, the CRS attributes are fed into NIP tomography resulting in a kinematically inverted velocity model and the source locations in depth. 




Figure 4: 2D velocity model used for the modeling of the considered passive seismic events. While the upper part is laterally homogeneous, the second interface reveals a finite curvature which results in lateral velocity variations in the deeper part. The locations of the passive seismic sources are represented by circles and their respective simulated excitation times are annotated. 

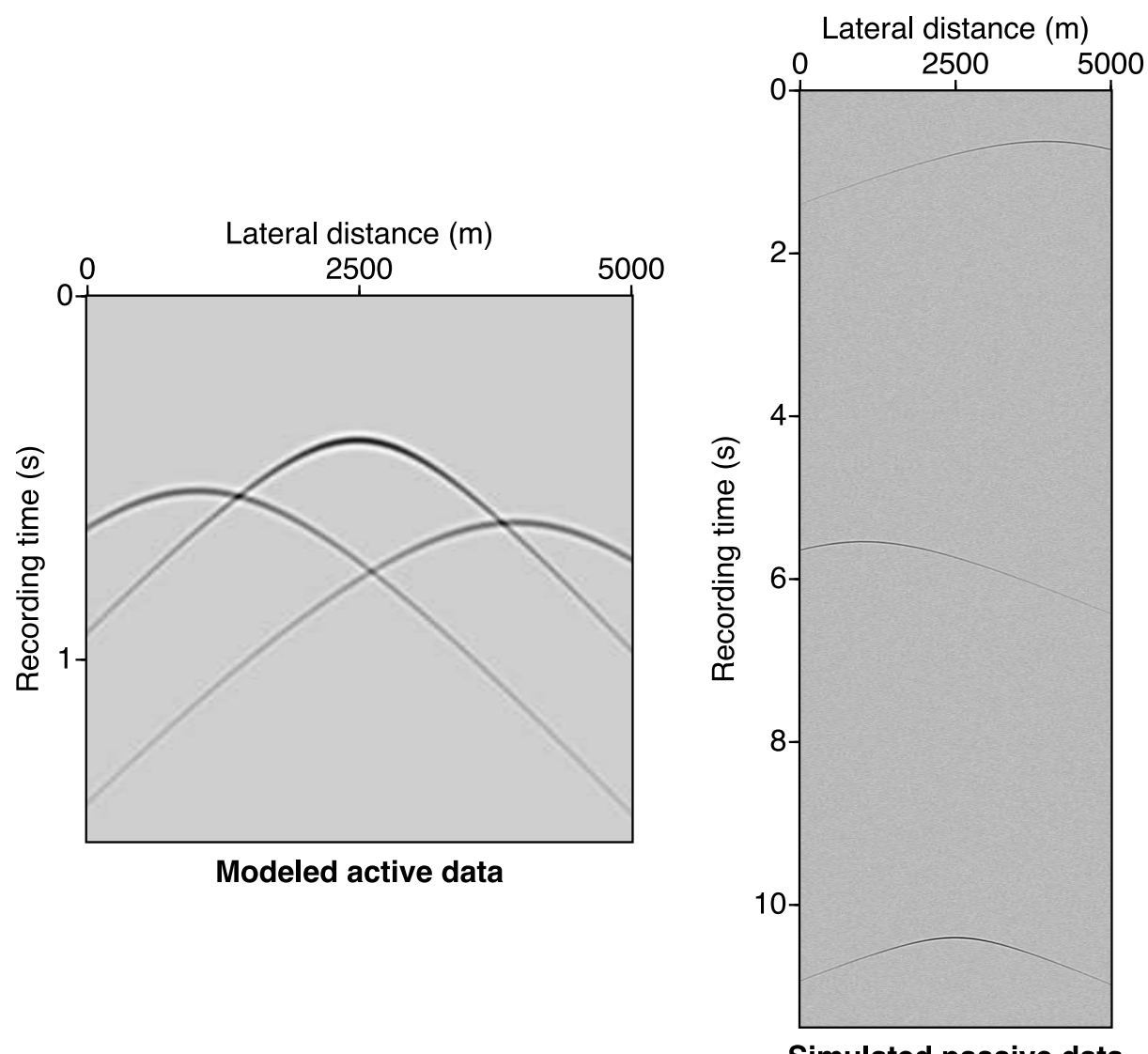

Figure 5: Synthetic passive seismic data, generated via ray modeling for the velocity distribution shown in Figure 4. To simulate a passive seismic experiment, the raw modeled events (left) were shifted in time by either 0,5 or $10 \mathrm{~s}$ (compare Figure 4) and random Gaussian noise with a signal-to-noise ratio of 5 was added (right). 


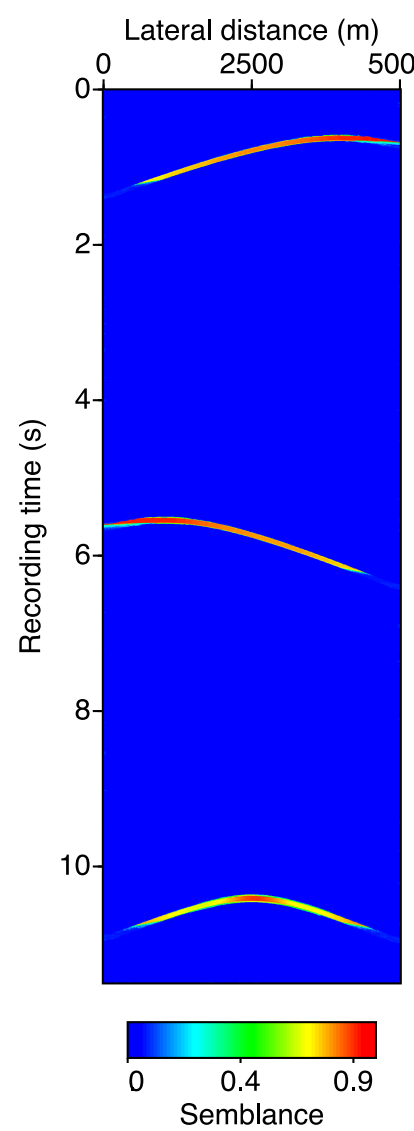

(a)



(b)

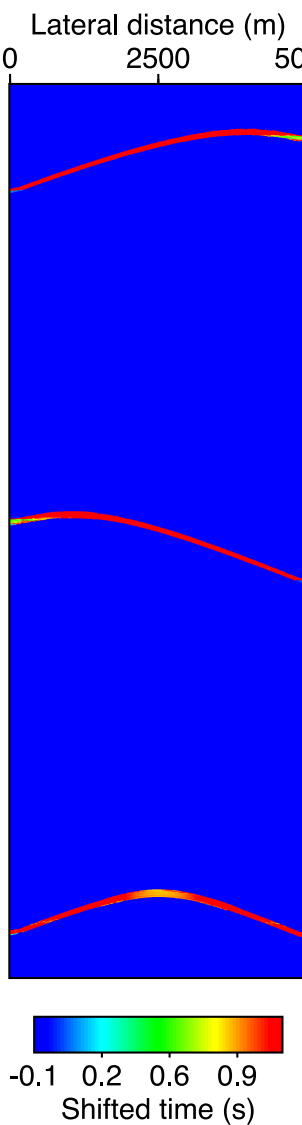

(c)

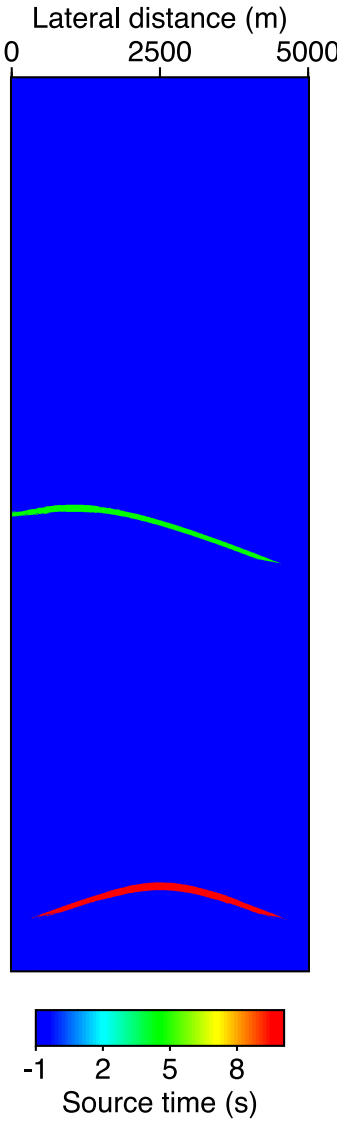

(d)

Figure 6: Attribute estimation results for the considered synthetic pssive experiment. Displayed are (a) the maximized semplance section, (b) the horizontal emerging slowness $p_{0 x}$, (c) the shifted reference time $t_{\text {shift }}$, and (d) the source excitation time. While the left three panels are also available in active seismic applications, the estimation results of the source time displayed on the right is a new ingredient in the passive seismic workflow and crucial for the subsequent localization in depth. 

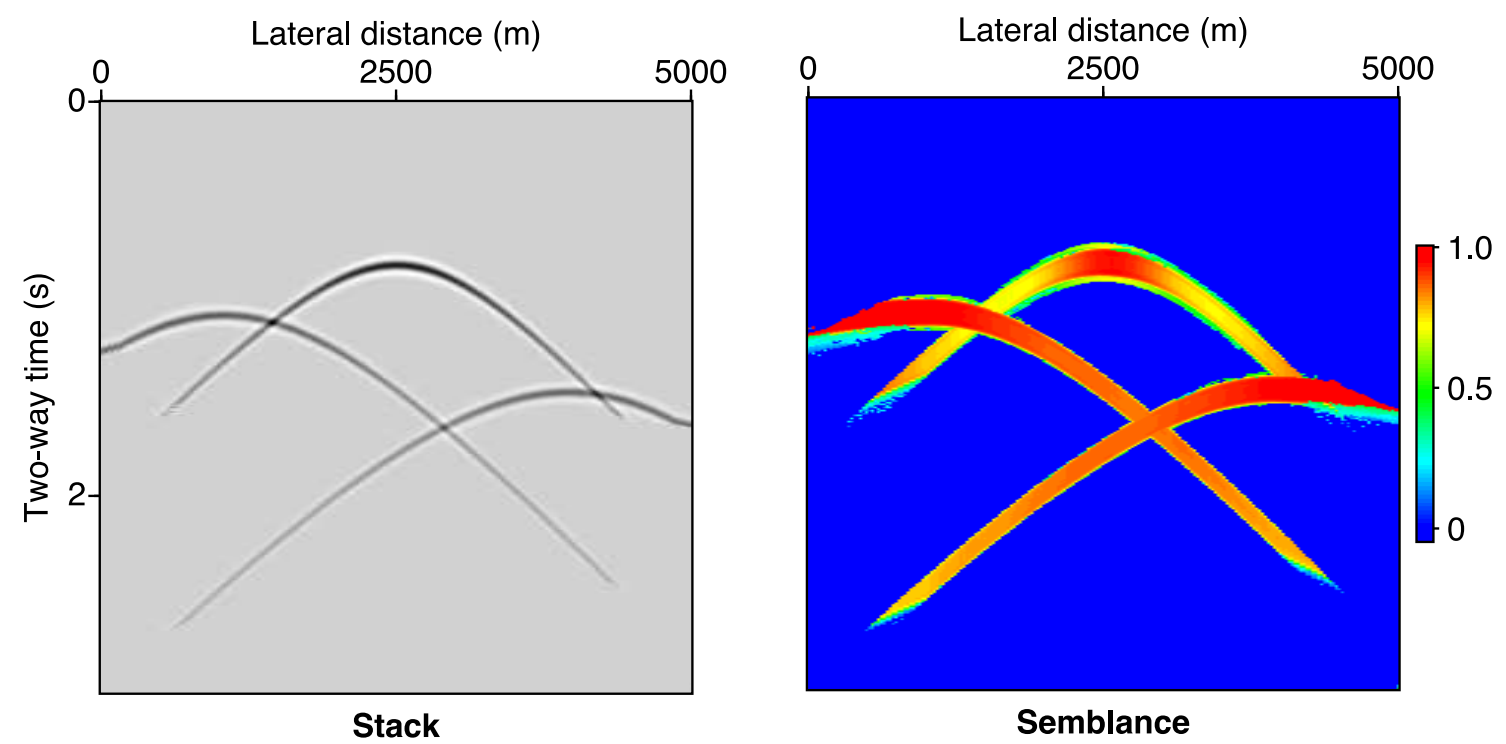

Semblance

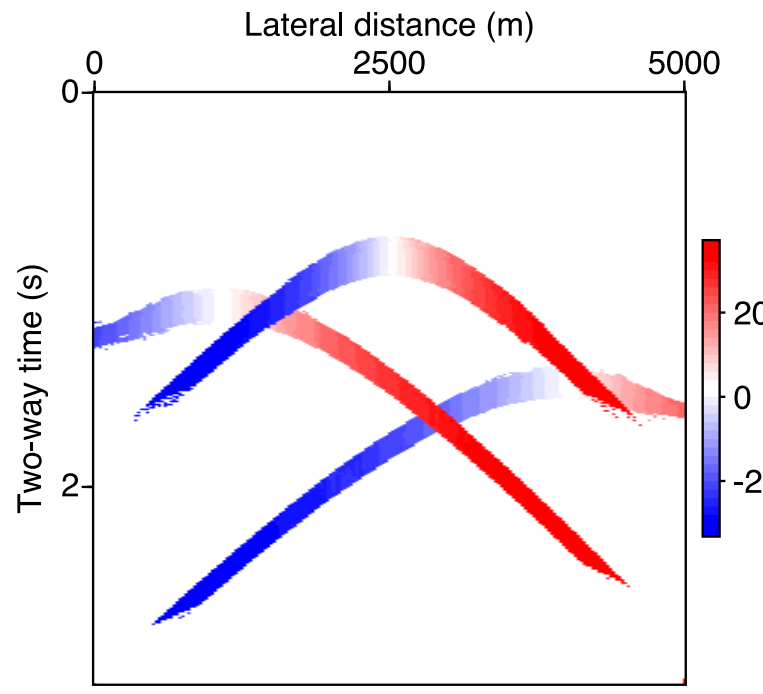

Emergence angle (deg)

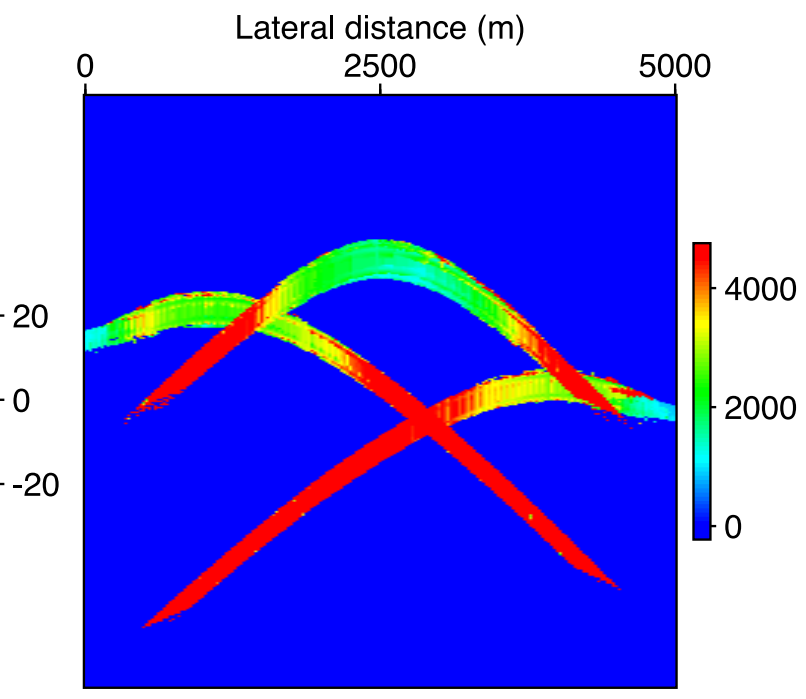

NIP wave radius $(m)$

Figure 7: Attribute sections used as input for NIP tomography, consisting of the optimized semblance, stack, emergence angle $\alpha_{0}$, and emerging radius of curvature of the NIP wave $R_{\mathrm{NIP}}$, the two latter translated from the horizontal slowness $p_{0 x}$ and the shifted reference time $t_{\text {shift }}$ after the correction for the individual source times $t_{s}$. 




(a)

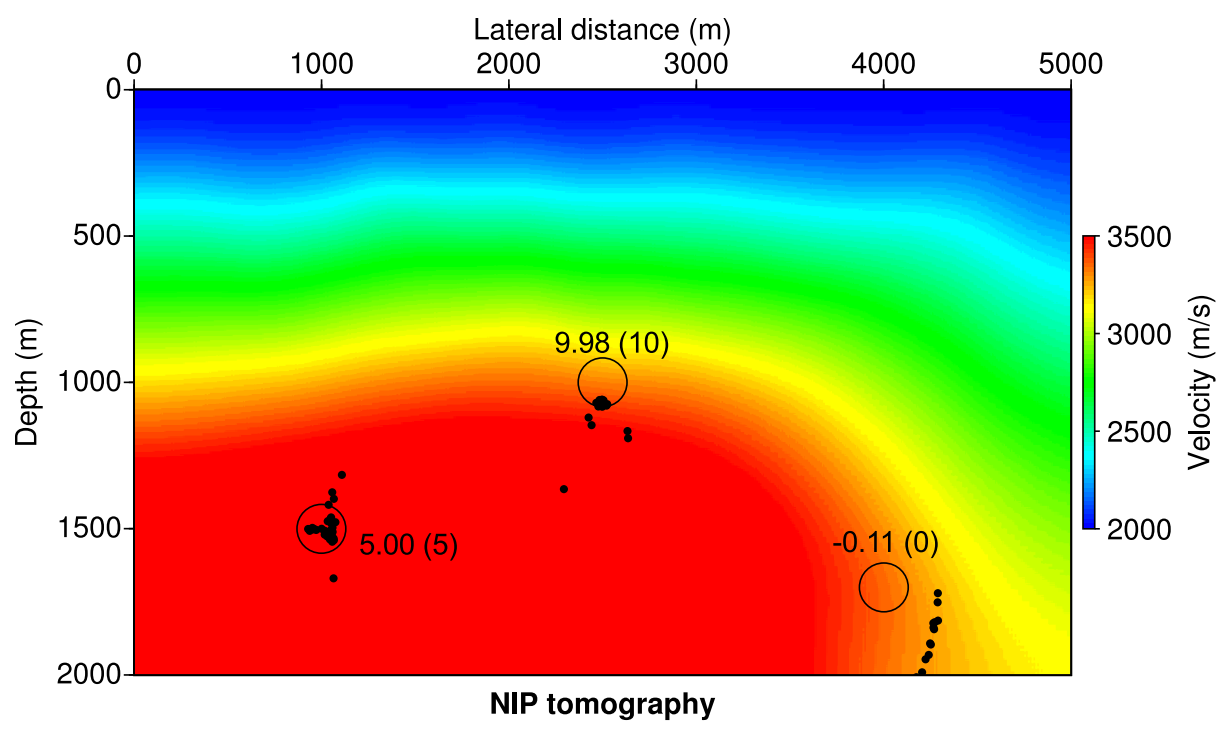

(b)

Figure 8: (a) Constant starting model with $v_{0}=2000 \mathrm{~m} / \mathrm{s}$ and (b) NIP tomographic inversion after 12 iterations. Again, like in Figure 8(a), the circles denote the true locations of the passive seismic sources and the black dots indicate the locally estimated depth locations of the passive seismic source. The respective estimated excitation times (shown in seconds) are in reasonable agreement with the exact solution (in brackets). 